\title{
Soluble IL-2Ra correlates with imbalances of Th1/Th2 and Tc1/Tc2 cells in patients with acute brucellosis
}

Hua-Li Sun ${ }^{1 \dagger}$, Cheng-Jie Ma ${ }^{2 \dagger}$, Xiu-Fang Du ${ }^{3}$, Si-Yuan Yang ${ }^{2}$, Xiao Lv ${ }^{4}$, Hong Zhao ${ }^{4}$, Ling-Hang Wang ${ }^{2}$, Yun-Xia Tang ${ }^{2}$, Xing-Wang Li ${ }^{5}$ and Rong-Meng Jiang ${ }^{5^{*}}$

\begin{abstract}
Background: Previous studies showed that soluble IL-2Ra is an important marker of cellular immune activation and might be a marker of treatment efficacy for children with brucellosis. However, data regarding adult patients with brucellosis were unknown. The aim of study was to explore the potential role of serum sIL-2Ra evaluating treatment responses in adult patients with brucellosis, and T cell immune status was also examined.

Methods: During January 2016-April 2017, 30 patients with acute brucellosis from the Third People's Hospital of Linfen in Shanxi Province and Beijing Di Tan Hospital, and 28 healthy controls were included in this study.

Peripheral blood samples were collected before and after six weeks of antibiotic treatment. Serum sIL-2Ra levels were measured by enzyme-linked immunosorbent assay, and the percentage of Th1, Th2, Tc1, Tc2, and Tregs was detected by flow cytometry after intracellular staining for cytokines (interferon- $\gamma$ and interleukin-4) and Foxp3 in T lymphocytes from peripheral blood. The obtained data were analyzed with Wilcoxon ranked sum tests for paired values, Mann-Whitney U-tests for comparisons between patients and healthy controls, and Spearman rank tests for correlation analyses.
\end{abstract}

Results: Serum SIL-2Ra levels were significantly higher in patients than in controls ( $P=0.001)$. A significant decline was observed in patients after the cessation of treatment $(P<0.001)$ and return to normal $(P>0.05)$. Th1, Tc1, Th2, and Tc2 cell frequencies were higher in patients than in healthy subjects $(P<0.05)$, while the Th1/Th2 and Tc1/Tc2 ratios were significantly lower ( $P=0.0305$ and 0.0005 , respectively) and returned to normal levels after treatment. In patients with acute brucellosis, serum sIL-2Ra levels were negatively correlated with the Th1/Th2 ratio $(r=-0.478$, $P=0.028)$, Tc1 $/ \mathrm{Tc} 2$ ratio $(r=-0.677, P=0.001)$, and Tc1 percentage $(r=-0.516, P=0.017)$. Serum slL-2Ra and Tc2 percentages were positively correlated $(r=0.442, P=0.045)$.

Conclusions: Based on the correlations with Th1/Th2 and Tc1/Tc2 ratios, serum slL-2Ra levels may reflect the immune response status. sIL-2Ra may be a marker for therapeutic efficacy in acute brucellosis.

Keywords: Brucellosis, slL-2Ra, Tc1, Tc2, Th1, Th2

\footnotetext{
*Correspondence: 13911900791@163.com

Hua-Li Sun and Cheng-Jie Ma share first authorship.

${ }^{5}$ Center for Infectious Diseases, Beijing Di Tan Hospital, Capital Medical

University, Beijing, China

Full list of author information is available at the end of the article
}

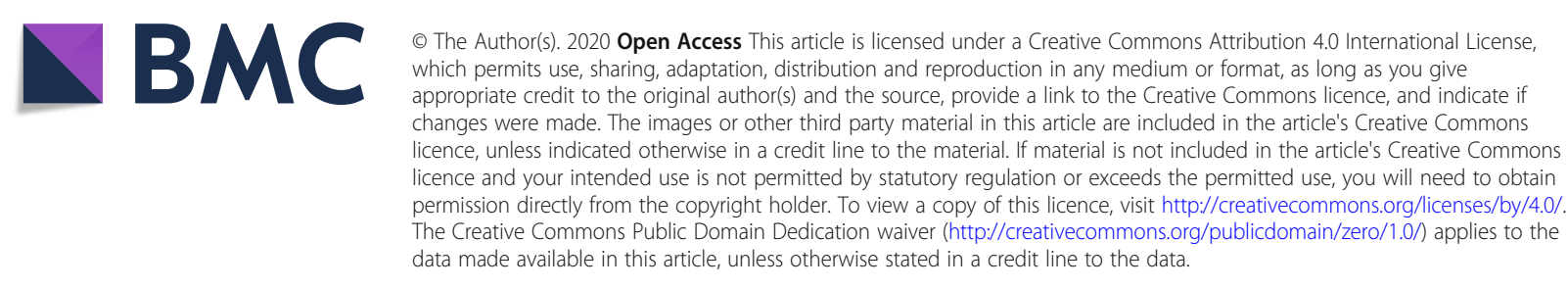




\section{Background}

Brucellosis, a re-emerging zoonotic infection worldwide, is a serious public health problem in many developing countries, with more than half a million new human infections each year [1]. The lack of an effective therapy or safe vaccine and tendencies towards chronicity and persistence [2] emphasize the importance of research focused on the immune response. Brucella is an intracellular pathogen that survives in macrophages. Host protection against Brucella depends on cell-mediated immunity, mainly involving activated antigen-presenting cells (macrophages and dendritic cells), CD4+ $\mathrm{T}$ helper cells (Th), and CD8+ cytotoxic T cells (Tc). The activation of monocyte macrophages and $\mathrm{T}$ lymphocytes induces a large number of immune molecules, including pro-inflammatory cytokines, anti-inflammatory cytokines, and chemokines, to coordinate the immune response, thereby determining disease progression and outcomes [3-6]. Studies have shown that mononuclear macrophages are activated with the release of soluble immunosuppressive molecules, such as SCD163 and sCD14, after Brucella infection, and changes in these immune molecules are expected to be effective indicators of treatment effects [7-9].

The soluble form of IL-2R $\alpha$ (sIL-2R $\alpha)$ is generated exclusively by the proteolytic cleavage of membrane IL$2 \mathrm{R} \alpha$ on activated $\mathrm{T}$ lymphocytes. IL-2R is composed of three subunits: $\alpha, \beta$, and $\gamma$, and the IL-2R $\alpha$ subunit is essential for high-affinity binding. sIL-2R $\alpha$ exerts immunoregulatory effects via the regulation of $\mathrm{T}$ cell responses [10-12]. An elevated concentration of sIL-2R $\alpha$ has been observed in autoimmune and inflammatory diseases [13], cancers [14], and various infectious diseases, including tuberculous [15-17]. Serum levels of sIL-2R $\alpha$ are useful markers of disease progression. In children with brucellosis, elevated sIL-2R $\alpha$ is associated with poor outcomes [18]. However, little is known about serum sIL-2R $\alpha$ levels in adults with brucellosis.

In this study, we measured serum sIL-2R $\alpha$ concentrations in adult patients with acute brucellosis before and after six weeks of antibiotic treatment to determine its prognostic value. It is well established that the Th1/Th2 and $\mathrm{Tc} 1 / \mathrm{Tc} 2$ ratios can reflect cellular responses. To provide a theoretical basis for the clinical application of sIL$2 \mathrm{R} \alpha$, we also investigated alterations in Th1/Tc1 lymphocytes (i.e., IFN- $\gamma$-producing lymphocytes) and Th2/Tc2 lymphocytes (i.e., IL-4-producing lymphocytes).

\section{Materials and methods}

\section{Characteristics of the study population}

In total, 30 patients with primary acute brucellosis (disease course $<6$ months) older than 18 years and 28 healthy volunteers from the same area, matched by sex and age, were included in this study. In the brucellosis group, the disease course of 12 patients (40\%) was less than 4 weeks, and the disease course of 18 patients (60\%) was between 4 and 8 weeks. All patients had been treated in the Department of Infectious Disease of the Third People's Hospital of Lin Fen in Shanxi Province and Beijing Di Tan Hospital between January 2016 and April 2017. Exclusion criteria for the healthy control subjects included acute/chronic diseases, smoking, medication, pregnancy, and any abnormalities in renal and liver function tests. Written informed consent was obtained from each subject and approval was obtained from the Ethics Committee of Beijing Di tan Hospital, Capital Medical University.

Brucellosis was diagnosed based on blood cultures and/or serologic tests according to current criteria [19]. Patients were excluded if they were pregnant, had evidence of auto-immune diseases, had other acute/chronic renal, liver, cardiovascular diseases, or received antibiotic treatment during the three months prior to the study. To avoid a Th2 bias in the immunologic studies, patients meeting the clinical criteria for atopy were excluded. Patients treated with immunosuppressants, immunomodulators, and/or other drugs capable of modifying the immune response during the three months preceding the study were also excluded.

All patients received the same antibiotic treatment regimen (ceftriaxone at $2 \mathrm{~g} /$ day for two weeks, doxycycline at $100 \mathrm{mg}$ twice daily for six weeks, and rifampin at 600-900 mg/day for six weeks).

The symptoms and signs of all patients improved substantially or disappeared after the 6-week antibiotic regimen, with good outcomes and no relapse at six months after the treatment ended.

\section{Sample collection}

Peripheral venous blood samples were collected before treatment and six weeks after treatment. The samples were stored in vacutainers (red top) without anticoagulant and EDTA-containing tubes and were used for serum and peripheral blood mononuclear cell (PBMC) isolation. The serum samples were obtained by centrifugation within four hours of blood collection and aliquots were stored at $-80{ }^{\circ} \mathrm{C}$ until use. PBMCs were isolated from EDTA blood by Ficoll-Paque (Amersham Pharmacia Biotech, Uppsala, Sweden) density gradient centrifugation. Cells were cryopreserved in fetal bovine serum (GIBCO, Grand Island, NY, USA) supplemented with $10 \%$ dimethylsulfoxide (DMSO) and stored in liquid nitrogen.

\section{Cytokine immunoassays}

Serum levels of sIL-2R $\alpha$ were measured in duplicate using a specific ELISA kit according to the manufacturer's instructions (R\&D Systems, Inc., Minneapolis, MN, USA). Serum samples require a 4-fold dilution 
prior to the assay. The minimum detectable doses of human sIL-2R $\alpha$ for the test kits are $10 \mathrm{pg} / \mathrm{ml}$, and the assay range is $78.0-5000 \mathrm{pg} / \mathrm{ml}$. This range can be broadened by the dilution of samples with high concentrations. All assays were performed and read at an Infectious Diseases Centre using Gen5 3.0 (BioTek Synergy H1, Winooski, VT, USA). A four-parameter logistic curve was used to interpolate sample values based on the standard curve at a wavelength of $450 \mathrm{~nm}$.

\section{Cell surface and intracellular cytokine staining}

Cryopreserved PBMCs were used to evaluate cell viability by cell surface and intracellular cytokine staining using samples from 21 patients and 28 healthy controls. Cryopreserved PBMCs were thawed in RPMI 1640 medium (Invitrogen, Carlsbad, CA, USA), washed with phosphatebuffered saline (PBS) containing 1\% bovine serum albumin (BSA), and incubated at room temperature for $20 \mathrm{~min}$ with the cell viability marker Fixable Viability Stain 510 (BD Biosciences, San Jose, CA, USA).

A lymphocyte phenotypic analysis was performed after staining with anti-CD4-PerCP-cy5.5 (BD Biosciences) and anti-CD8-APC-H7 antibodies. Intracellular IFN- $\gamma$ and IL-4 staining was performed after stimulation with a leukocyte activation cocktail with BD Golgi Plug (BD Biosciences) for $5 \mathrm{~h}$. The leukocyte activation cocktail contained PMA, ionomycin, and brefeldin A. Cells were initially stained with anti-CD4-PerCP-cy5.5 and antiCD8-APC-H7, fixed, and permeabilized with Cytofix/ Cytoperm according to the manufacturer's instructions (BD Biosciences), followed by staining with anti-IFN- $\gamma$ FITC (BD Biosciences) and anti-IL-4-PE. To identify Tregs, freeze-thawed PBMCs were stained for surface molecules with anti-CD4-PerCP-cy5.5 and anti-CD25$\mathrm{PE}-\mathrm{Cy} 7$ antibodies and for intracellular molecules with an anti-FoxP3-AF647 antibody, in accordance with the manufacturer's instructions (BD Biosciences).

All expression analyses were performed by flow cytometry using BD FACS Canto II with Diva (BD Biosciences). Forward scatter and side scatter light gating were used to exclude cell debris. Forward height and forward areas were used to exclude doublet cells. The final analysis was performed using FlowJo 10.0.7 (Tree Star Inc., Ashland, OR, USA).

\section{Statistical analyses}

Statistical analyses and graphical presentation of results were performed using Graph Pad Prism 7.0 (San Diego, CA, USA). Data are expressed as median values and analyzed using Wilcoxon ranked sum tests for paired values (i.e., pre- and post-treatment for the same subject) or Mann-Whitney U-tests for between subject comparisons (i.e., patients versus healthy controls). Correlations were evaluated using the Spearman test. All tests were two- tailed, and $P$-values of less than 0.05 were considered statistically significant.

\section{Results \\ Demographic, clinical, laboratory, and treatment data}

A total of 30 patients (24 males [80\%]) with acute brucellosis were enrolled, with an average age of 48 years (range: 20-69 years). We also enrolled 28 healthy controls (24 males [85.7\%]), with an average age of 46 years (range: 24-69 years). There was no significant difference between patients and healthy controls with respect to gender or age $(P>0.05)$. With regards to the diagnosis of brucellosis, 16 patients $(53.3 \%)$ had positive blood culture, the positive detection rate for the Rose-Bengal test was $100 \%$, and the serum agglutination titers were more than $1 / 160$ for all included patients. Common clinical symptoms were fever (83.3\%), sweating (83.3\%), weakness (76.7\%), arthralgia and myalgia (53.3\%), and anorexia (33.3\%). Traditional inflammatory markers including C-reactive protein (CRP) and erythrocyte sedimentation rates (ESR) were also assayed by standard laboratory methods. In patients with acute brucellosis, CRP and ESR levels were significantly increased (Table S1). After the termination of standard treatment, symptoms and signs gradually disappeared, and CRP and ESR levels returned to normal and did not reoccur after six months of follow-up.

Increased serum sIL-2Ra levels in patients with brucellosis decline upon treatment

Serum sIL-2R levels in patients with acute brucellosis and in healthy controls are summarized in Fig. 1. The median (Q1-Q4) level of sIL-2R $\alpha$ was 1807.14 (1104.66-2870.97) $\mathrm{pg} / \mathrm{ml}$ in the pre-treatment group, $864.07(630.82-1414.97) \mathrm{pg} / \mathrm{ml}$ in the post-treatment group, and $739.15(528.41-1104.59) \mathrm{pg} / \mathrm{ml}$ in healthy controls. Median sIL-2R $\alpha$ levels were significantly higher in patients with acute brucellosis than in healthy controls (Fig. $1, P=0.001$ ). After six weeks of treatment, serum sIL-2R $\alpha$ levels decreased significantly (Fig. $1, P<$ 0.0001 ) and recovered to the levels in healthy controls (Fig. 1, $P>0.05$ ).

Increased percentages of Th1, Th2, Tc1, and Tc2 cells, and decreased Th1/Th2 and Tc1/Tc2 ratio in patients with brucellosis

To investigate changes in CD4+ and CD8+ T cell subsets in patients with acute brucellosis, we analyzed the following subsets in the blood: CD4 + IFN- $\gamma+(\mathrm{Th} 1), \mathrm{CD} 4+\mathrm{IL}-$ $4+(\mathrm{Th} 2), \mathrm{CD} 8+\mathrm{IFN}-\gamma+(\mathrm{Tc} 1)$, and CD8 + IL-4+ (Tc2) (Fig. 2 and Table 1). Lymphocytes were gated based on a forward vs side scatter dot plot, and then, we analyzed different $\mathrm{CD} 4+$ and $\mathrm{CD} 8+\mathrm{T}$ cell subsets based on gated cells. A representative example of the gating strategy is illustrated in Figure S1. Peripheral blood lymphocytes from 


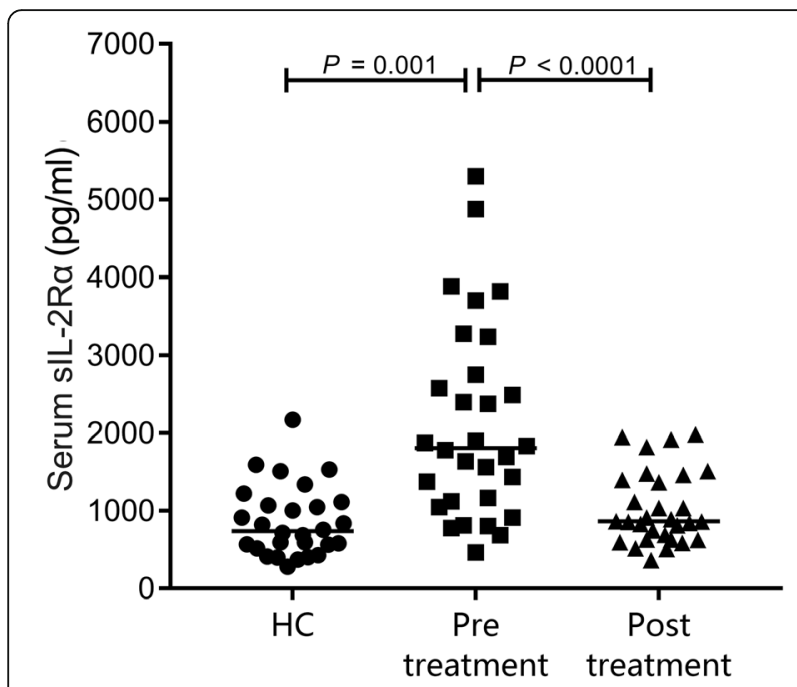

Fig. 1 Serum sIL-2Ra levels for patients with acute brucellosis (preand post-treatment) and healthy controls. Circulating levels of sIL-2Ra in serum samples of patients with brucellosis (pre- and post-treatment, $n=30$ ) compared to levels in healthy controls $(n=28)$, represented by dots. The horizontal line represents the median. A Mann-Whitney Utest was performed to evaluate the difference between controls and patients. Pre- and post-treatment levels were compared by the Wilcoxon matched-pair signed-rank test. sIL-2Ra: soluble Interleukin-2 receptor $\mathrm{a}$; $\mathrm{HC}$ : healthy control

21 patients with brucellosis and 28 healthy controls were evaluated. As shown in Fig. 2a, b and Table 1, percentages of Th1 (14.1-29.4\%) and Th2 (0.6-1.2\%) were both markedly higher in patients than in healthy controls $(7.0-20.4 \%$ and $0.2-0.6 \%$, respectively; $P=0.0437$ and $P<0.0001$, respectively). Similar to Th1 and Th2 cells, the Tc1 (36.3$61.0 \%)$ and Tc2 percentages (1.5-2.5\%) were both significantly higher in patients than in controls $(26.5-51.2 \%$ and $0.5-1.3 \%$, respectively) (Table 1 and Fig. 2c, d; $P=0.0271$ and $P<0.0001$, respectively). However, further analyses indicated that the Th1/Th2 and Tc1/Tc2 ratios were significantly lower in patients than in healthy controls (Table 1; $P=0.0305$ and $P=0.0005$, respectively), and there was a significant bias toward both Th2 and Tc2 cell responses in patients with acute brucellosis. At the end of the treatment period, both Th1/Th2 and Tc1/Tc2 ratios returned to normal levels (Table $1 ; P>0.05$ ). Furthermore, as Treg cells with negatively regulatory effects on immunity, CD4 + CD25 + FoxP3 + T cells were evaluated. As shown in Table 1, although the proportion of Tregs was higher in patients, the difference between groups was not significant.

\section{Correlation between serum sIL-2Ra levels and the percentage of Th1, Th2, Tc1, and Tc2 cells in patients with brucellosis}

For a more detailed analysis, we explored the correlations between serum sIL-2R $\alpha$ levels and cell subsets
(Table 2 and Fig. 3). Serum sIL-2R $\alpha$ levels were inversely associated with the percentage of Tc1 cells (Fig. 3a; $r=$ $-0.516, P=0.017$ ), Th1/Th2 ratio (Fig. 3c; $r=-0.478$, $P=0.028$ ), and Tc1/Tc2 ratio (Fig. $3 \mathrm{~d} ; r=-0.677, P=$ 0.001 ) in patients with acute brucellosis. In contrast, serum sIL-2R $\alpha$ levels were positively correlated with the percentage of Tc2 cells (Fig. 3b; $r=0.442, P=0.045$ ). At the end of treatment period, the relationships disappeared, with normalized sIL-2R $\alpha$ levels. Surprisingly, no correlation was found between serum sIL-2 levels and proportions of Tregs.

\section{Discussion}

We investigated serum levels of the immune-inhibitory molecule sIL-2R $\alpha$, frequencies of Th1, Th2, and cytotoxic (Tc1 and Tc2) T-lymphocyte subsets in PMA and ionomycin-stimulated PBMCs as well as the frequency of Tregs in adult patients with brucellosis before and after the standard 6-week course of antibiotics. Our data agree with previous results showing that sIL-2R $\alpha$ levels are increased in patients with brucellosis. Furthermore, serum sIL-2R $\alpha$ levels were inversely related to the Th1/ Th2 ratio (especially the $\mathrm{Tc} 1 / \mathrm{Tc} 2$ ratio) during the acute phase of adult brucellosis, suggesting that serum sIL$2 \mathrm{R} \alpha$ could serve as an indicator of the immune state in Brucella infection. After effective treatment, serum sIL2 R $\alpha$ levels and Th1/Th2 and Tc1/Tc2 ratios returned to normal, indicating an improvement in host resistance. These findings further suggest that serum sIL-2R $\alpha$ is a potentially effective marker of treatment responses in adult patients with brucellosis.

In a murine model, protective acquired immunity to Brucella involves the $\mathrm{T}$ cell-dependent activation of macrophages involving both CD4+ and CD8 + T cells. It is well established that the clearance of Brucella requires IFN- $\gamma$-mediated type- $1 \mathrm{~T}$ cell (Th1 and Tc1) immune responses [20-22], and the IL-4, IL-10, Th2 response determines susceptibility to Brucella and disease severity [23-25]. As another T-cell subset, Tregs have a pivotal role in the regulation of the immune response to Brucella. An in vivo experiment demonstrated that the production of IL- 10 by CD $4+$ CD $25+T$ cells is critical for the modulation of macrophage function during early Brucella infections [26]. In a BALB/c mouse model of brucellosis, CD $4+\mathrm{CD} 25+\mathrm{T}$ regulatory cells may limit the effectiveness of $\mathrm{CD} 4+\mathrm{T}$ cells and favor the maintenance and progression of B. abortus infection [27].

IL-2R $\alpha$ is expressed on many hematopoietic cells, including subsets of $\mathrm{T}$ and $\mathrm{B}$ cells (e.g., regulatory $\mathrm{T}$ cells), and is upregulated by the activation of these cells. Upon activation, immune cells proliferate and release sIL-2R $\alpha$ in vitro $[28,29]$. Therefore, measurable serum sIL-2R $\alpha$ levels are thought to reflect sustained immune activation during infection or inflammation and have been used as 


\section{a}

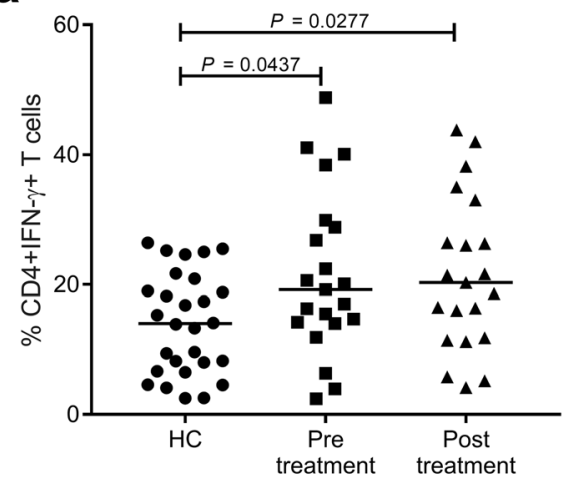

C

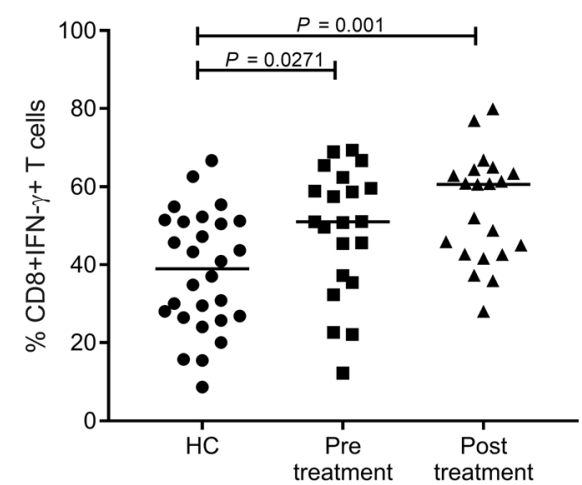

b

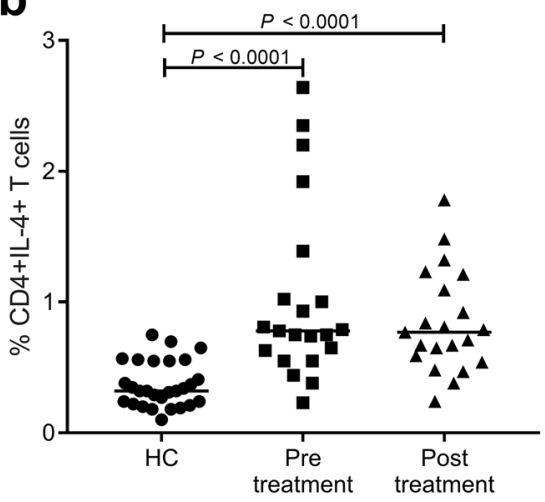

d

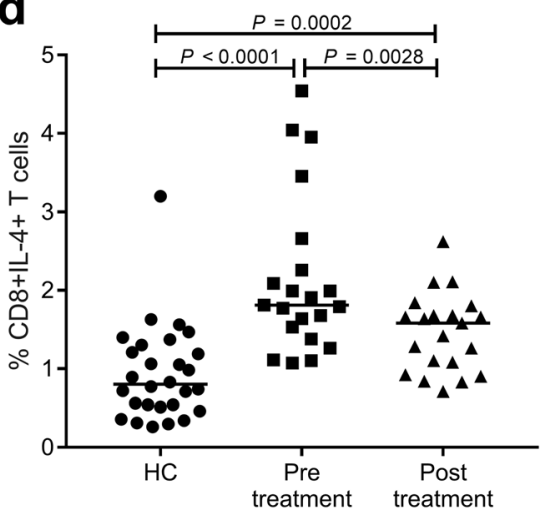

Fig. 2 Percentages of Th1, Th2, Tc1, and Tc2 in samples from patients with acute brucellosis pre-and post-treatment and healthy controls. a, b, $\mathbf{c}$ and $\mathbf{d}$ Comparisons of the percentages of Th1, Th2, Tc1, and Tc2 cells (Th1: CD4 + IFN- + + T cells, Th2: CD4 + IL-4 + T cells, Tc1: CD8 + IFN- - + T cells, Tc2: CD8 + IL-4 + T cells) in healthy controls (HC) and patients with acute disease before treatment (Pre-treatment) and after treatment (Posttreatment) ( $n=28,21$, and 21, respectively) are shown. The vertical line represents the median. A Mann-Whitney U-test was performed to compare controls and patients, and pre- and post-treatment values for patients were compared by the Wilcoxon matched-pair signed-rank test. HC: healthy control

Table 1 Percentages of Th1, Th2, Tc1, and Tc2 from acute brucellosis samples pre-and post-treatment and healthy controls

\begin{tabular}{|c|c|c|c|c|}
\hline & $\begin{array}{l}\text { Pre-treatment } \\
(n=21) \\
\text { Median (Q1-Q3) }\end{array}$ & $\begin{array}{l}\text { Post-treatment } \\
(n=21) \\
\text { Median (Q1-Q3) }\end{array}$ & $\begin{array}{l}\text { Healthy control } \\
(n=28) \\
\text { Median }(\mathrm{Q} 1-\mathrm{Q} 3)^{\mathrm{f}}\end{array}$ & $\begin{array}{l}P \text {-value }{ }^{a} \text { pre- vs } \\
\text { post-treatment }\end{array}$ \\
\hline $\mathrm{CD} 4+\mathrm{IFN}-\gamma+$ & $19.2(14.1-29.4) *$ & $20.3(11.6-29.7) *$ & $14.0(7.0-20.4)$ & $n s^{b}$ \\
\hline CD4 + IL-4+ & $0.8(0.6-1.2) *$ & $0.8(0.6-1.2) *$ & $0.3(0.2-0.6)$ & ns \\
\hline $\mathrm{CD} 8+\mathrm{IFN}-\gamma+$ & $51.0(36.3-61.0) *$ & $60.6(42.7-63.9) *$ & $39.0(26.5-51.2)$ & ns \\
\hline CD8 + IL-4+ & $1.8(1.5-2.5) *$ & $1.6(1.0-1.7) *$ & $0.8(0.5-1.3)$ & 0.0028 \\
\hline Treg $^{c}$ & $1.4(0.9-2.1)$ & $2.0(1.2-2.5)$ & $1.2(0.8-2.2)$ & 0.093 \\
\hline Th1/Th2 ratio ${ }^{d}$ & $22.6(15.8-28.7)^{*}$ & $23.8(18.4-32.7)$ & $37.4(19.2-71.4)$ & ns \\
\hline Tc1/Tc2 ratio ${ }^{e}$ & $30.4(14.1-35.0) *$ & $39.0(30.5-45.4)$ & 50.7 (31.0-73.7) & 0.0006 \\
\hline
\end{tabular}

${ }^{\mathrm{a}}$ Data was analyzed using Wilcoxon matched-pair signed-rank test

${ }^{\mathrm{b}} \mathrm{ns}$, no significant difference

${ }^{\mathrm{C}}$ Treg, proportion of $\mathrm{CD}^{+}{ }^{+} \mathrm{CD} 25^{+} \mathrm{FoxP}^{+}{ }^{+} \mathrm{T}$ cells among $\mathrm{CD} 4^{+}$cells

${ }^{\mathrm{d}}$ Th1/Th2 ratio, ratio of peripheral CD4 + T cells producing IFN- $\gamma(\mathrm{CD} 4+\mathrm{IFN}-\gamma+)$ to those producing IL-4 (CD4 + IL-4+)

${ }^{\mathrm{e}} \mathrm{Tc} 1 / \mathrm{Tc} 2$ ratio, ratio of peripheral CD8 $+\mathrm{T}$ cells producing IFN- $\gamma(\mathrm{CD} 8+\mathrm{IFN}-\gamma+)$ to those producing IL-4 (CD8 + IL-4+)

${ }^{f} \mathrm{Q} 1$ and Q3 represent the first and third quartiles, respectively

*Significant difference $(p<0.05)$ in comparison with healthy controls 
Table 2 Correlations between serum sIL-2Ra levels and percentages of Th1, Th2, Tc1 and Tc2 in acute adult brucellosis $(n=21)$

\begin{tabular}{lll}
\hline & $R$ & $P$-value \\
\hline CD4 + IFN- $\gamma+$ & -0.343 & 0.128 \\
CD4 + IL-4+ & 0.001 & 0.996 \\
CD8 + IFN- $\gamma+$ & -0.517 & 0.017 \\
CD8 + IL-4+ & 0.442 & 0.045 \\
CD4 + CD25 + FoxP3 + T cell & -0.208 & 0.366 \\
Th1/Th2 ratio & -0.478 & 0.028 \\
Tc1/Tc2 ratio & -0.677 & 0.001 \\
\hline
\end{tabular}

$R$, Spearman correlation coefficient

Th1, CD4 + IFN- $\gamma+$; Th2, CD4 + IL-4+; Tc1, CD8 + IFN- $\gamma+$; Tc2, CD8 + IL-4+

a biomarker to characterize disease progression, prognosis, and treatment. In a previous study of 20 children with brucellosis [18], increases sIL-2R $\alpha$ levels were reduced after the 6-week antibiotic regimen in 17 children who subsequently had good outcomes without relapse. In three patients with relapse, serum sIL-2R $\alpha$ levels did not decline significantly after the termination of treatment. Our sIL-2R $\alpha$ assay results for adults confirmed previous observations that SIL-2R $\alpha$ is significantly elevated in patients with brucellosis. However, our data showed that serum sIL-2R $\alpha$ levels return to normal at the end of the treatment period. The differences between studies may be explained by differences in medications; in our study, the regimen was doxycycline and rifampin for six weeks plus ceftriaxone for two weeks, while the previous study used a combination of oral rifampin and trimethoprim-sulfamethoxazole for six weeks. Unfortunately, due to the small sample size, we did not capture relapses, which would weaken the importance of sIL$2 \mathrm{R} \alpha$ as a prognostic marker in adult patients with brucellosis.

An imbalance between the production of Th1 and Th2 (Tc1 and Tc2) cytokines contributes to an impairment in $\mathrm{T}$ cell-mediated immunity, which may ultimately influence anti-bacterial immune responses. In our study, we observed imbalances between Th1 and Th2 as well as between Tc1 and Tc2, which have been reported with various intracellular bacterial infections, including tuberculosis [30, 31]. Our findings revealed higher
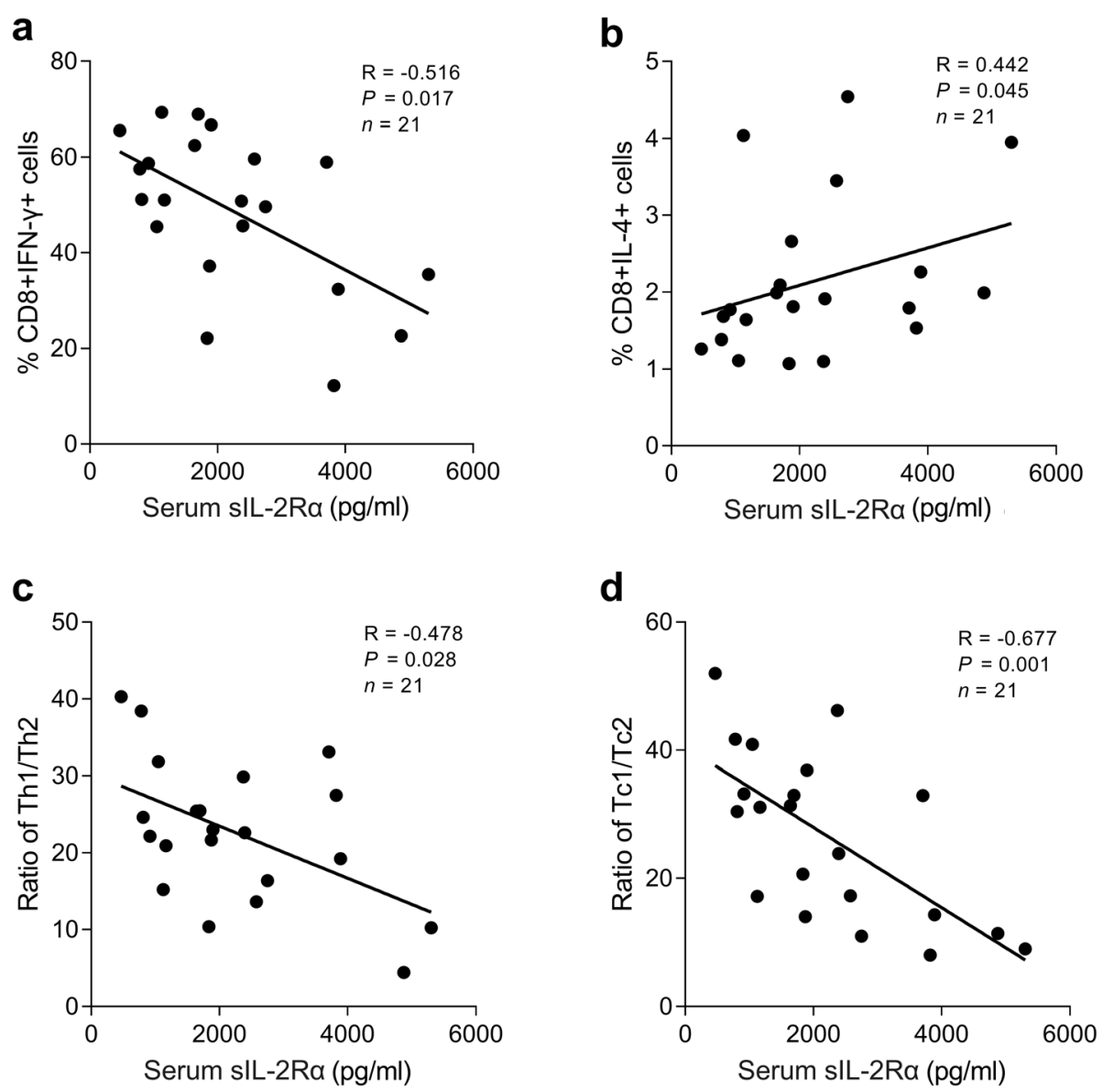

Fig. 3 Correlations between serum slL-2Ra levels and the percentage of Tc cells, Th1/Th2 ratio, and Tc1/Tc2 ratio in patients with brucellosis ( $n=21$ ) 
percentages of Th1, Tc1, Th2, and Tc2 lymphocytes in patients with brucellosis as compared than in healthy controls, which consistent with previous results [32]. Type 1 and type 2 immune responses are induced simultaneously in the acute phase. Our results also indicated that the Th1/Th2 and Tc1/Tc2 ratios are significantly decreased in the acute phase, reflecting a general skew in the immune response away from type- 1 and toward type-2, which is associated with the suppression of the immune response; this supports the concept that a predominantly type- 2 cell response is associated with the pathogenesis of brucellosis. However, Rafiei et al. found that the Th1 response is dominant in early brucellosis, with a shift toward the Th2 phenotype as the disease progresses [33]. This difference among studies might be due to the difference in patient recruitment, since the previous study included patients who had already received antibiotic treatment. In the early stage of infection in mouse models, $B$. abortus induces the expression of low levels of proinflammatory cytokines and high levels of anti-inflammatory cytokines [26, 34, 35]. Our findings, in conjunction with previous investigations, suggest that immunological changes in Th1/Th2 or Tc1/ Tc2 ratios may promote an immunosuppressive condition in patients with acute brucellosis and a limited inflammatory response develops in the early phase.

To further clarify the immunological basis of sIL-2R $\alpha$ as a prognostic marker, we analyzed the correlation between serum sIL-2R $\alpha$ levels and T cell immune parameters in patients with acute brucellosis. We found the first evidence for negative correlations between serum sIL2R $\alpha$ levels and Th1/Th2 and Tc1/Tc2 ratios, supporting the immunosuppressive effect of sIL-2R $\alpha$ during Brucella infection. Interestingly, serum sIL-2R $\alpha$ levels were only associated with Tc1 and Tc2 cell frequencies, but not $\mathrm{CD} 4+\mathrm{T}$ cells. At the end of the treatment period, the serum sIL-2R $\alpha$ levels, Th1/Th2 ratio, and Tc1/Tc2 ratio return to normal. These results establish several important points. First, serum sIL-2R $\alpha$ levels might reflect peripheral blood T-cell profiles and could serve as an indicator of the immune state, which is closely related to prognosis. Second, the strong correlation between serum sIL-2R $\alpha$ levels and CD8 + T cell subsets, but not $\mathrm{CD} 4+\mathrm{T}$ cells, implied that $\mathrm{CD} 8+\mathrm{T}$ cells play a more prominent role in the control of Brucella infection. Recent studies have shown that defects in Brucella-responsive $\mathrm{CD} 8(+) \mathrm{T}$ cells allow the persistence of infection $[21,22,36]$. Therefore, we speculated that serum sIL$2 \mathrm{R} \alpha$ levels contribute to the down-regulation of CD8 + T cells in patients with brucellosis. Although a detailed functional analysis of the role in sIL-2R $\alpha$ in regulating the immune response in vitro is still needed, several recent studies have made progress in our understanding of the sIL-2R $\alpha$-mediated suppressive mechanism. In lymphoma, sIL-2R $\alpha$ may present IL-2 in vitro to CD4 + $\mathrm{T}$ cells and cause the differentiation of these cells into FoxP3 + Treg cells, resulting in the suppression of CD8 + $\mathrm{T}$ cells [11]. We also study changes in Treg (CD4+ $\mathrm{CD} 25+$ FoxP3 + T) cells and did not detect a correlation between Treg cells and sIL-2R $\alpha$. Hence, the specific immunological mechanism of serum sIL-2R $\alpha$ requires further study. An unexpected discovery was that although the percentage of Treg cells was elevated in patients, there was no significant difference between patients and healthy controls, different from the results of a previous study showing that Treg cells are increased in patients and decline with therapy [37]. The sample size, patient characteristics, and antibiotic may explain the difference.

Despite the meaningful observations, our study has some limitations, including the small sample size and few time points measured. The prognosis of brucellosis was correlated with clinical staging; research with a larger sample size, more follow-up time points, and analyses from different clinical stages will help to better explain sIL-2R $\alpha$ as a beneficial laboratory test. Additionally, the present study only showed an association between sIL-2R $\alpha$ and $\mathrm{CD} 8+\mathrm{T}$ cell subpopulations in acute patients with brucellosis, and mechanistic research involving sIL-2R $\alpha$-mediated immune suppression is needed to better characterize the relationship between sIL-2R $\alpha$ and $\mathrm{CD} 8+\mathrm{T}$ cell immunity.

\section{Conclusions}

These data demonstrate that serum sIL-2R $\alpha$ levels increase during acute infection but return to normal levels during recovery. There is a strong negative correlation between increased serum sIL-2R $\alpha$ levels and the $\mathrm{Tc} 1 / \mathrm{Tc} 2$ ratio during the acute phase. Based on these results, serum sIL-2R $\alpha$ levels could serve as an indicator of the immune suppressive state in Brucella infection and is a candidate marker of treatment responses in adult patients with brucellosis. Further investigations with a larger sample sizes and longer follow-up to capture relapses will strengthen the clinical value of sIL-2R $\alpha$.

\section{Supplementary information}

Supplementary information accompanies this paper at https://doi.org/10. 1186/s40249-020-00699-y.

Additional file 1: Figure S1. Representative gating strategy for different CD4+ and CD8 + T cell subsets. In this sample gating, cells were first gated for lymphocytes (SSC-A vs FSC-A). The lymphocytes were gated based on single cells and live cells and were further analyzed for IFN- $\gamma$-positive, IL-4 CD4 + T, and CD8 + T cells.

Additional file 2: Table S1. Serum sIL-2Ra and CRP, ESR levels of patients with acute brucellosis in pre-and post-treatment and healthy controls. 


\section{Abbreviations}

ELISA: Enzyme-linked immunosorbent assay; IFN- $\gamma$ : Interferon- $\gamma$; IL4: Interleukin-4; Th: T helper cells; Tc: CD8+ cytotoxic T cells; sIL-2Ra: Soluble Interleukin-2 receptor a; PBMC: Peripheral Blood mononuclear cell; PBS: Phosphate-buffered saline; BSA: Bovine serum albumin; CRP: C-reactive protein; ESR: Erythrocyte sedimentation rate; DMSO: Dimethylsulfoxide

\section{Acknowledgements}

The authors are grateful to technicians from the clinical laboratory and medical staff from the Department of Infectious Diseases of Linfen City in Shanxi Province for blood sample collection as well as to all of the healthy controls. We would like to thank Editage (www.editage.cn) for English language editing.

\section{Authors' contributions}

HL-S, CJ-M and RM-J contributed to study design, drafting of the manuscript transcript. HL-S, XF-D, X-L, and $H-Z$ contributed to samples collection and transportation. HL-S, LH-W, YX-T and SY-Y contributed to laboratory testing. HL-S, CJ-M, JRM and XW-L gathered and analyzed the data. All authors read and approved the final manuscript

\section{Funding}

This study was supported by Beijing Municipal Administration of Hospitals Incubating Program (code: PX2016019), Beijing Di Tan Hospital Fund (Grant No. DTQL201404) and Beijing Municipal Commission of Health and Family Planning Culture Project of Advanced Intellectuals (code: 2015-3-109).

\section{Availability of data and materials}

Not applicable.

\section{Ethics approval and consent to participate}

Written informed consent was obtained from each subject and approval was obtained from the Ethics Committee of Beijing Di tan Hospital, Capital Medical University.

\section{Consent for publication}

Not applicable.

\section{Competing interests}

The authors declare that they have no competing interests.

\section{Author details}

${ }^{1}$ Department of Infectious Diseases, The Affiliated Hospital of Qingdao University, Qingdao, Shandong, China. ${ }^{2}$ The Laboratory of Infectious Diseases Centre, Beijing Di Tan Hospital, Capital Medical University, Beijing, China. ${ }^{3}$ Department of Infectious Diseases, the Third People Hospital, Linfen City, Shanxi Province, China. ${ }^{4}$ Department of Laboratory Medicine, the Third People Hospital, Linfen City, Shanxi Province, China. ${ }^{5}$ Center for Infectious Diseases, Beijing Di Tan Hospital, Capital Medical University, Beijing, China

\section{Received: 11 March 2020 Accepted: 11 June 2020}

Published online: 13 July 2020

\section{References}

1. Pappas G, Papadimitriou P, Akritidis N, Christou L, Tsianos EV. The new global map of human brucellosis. Lancet Infect Dis. 2006;6:91-9.

2. Franco MP, Mulder M, Gilman RH, Smits HL. Human brucellosis. Lancet Infect Dis. 2007:7:775-86.

3. Baldwin $\mathrm{CL}$, Parent M. Fundamentals of host immune response against Brucella abortus: what the mouse model has revealed about control of infection. Vet Microbiol. 2002;90:367-82.

4. Skendros P, Pappas G, Boura P. Cell-mediated immunity in human brucellosis. Microbes Infect. 2011;13:134-42.

5. de Figueiredo P, Ficht TA, Rice-Ficht A, Rossetti CA, Adams LG. Pathogenesis and immunobiology of brucellosis: review of Brucella-host interactions. Am J Pathol. 2015;185:1505-17.

6. Byndloss MX, Tsolis RM. Brucella spp. virulence factors and immunity. Annu Rev Anim Biosci. 2016;4:111-27.

7. Ayaslioglu E, Tekeli E, Birengel S. Significant elevation of serum soluble CD14 levels in patients with brucellosis. Jpn J Infect Dis. 2005;58:11-4.
8. Ayarcı AO, Yılmaz E, Sığılı D, Budak F, Göral G, Oral HB. Diagnostic value of serum concentrations of high-mobility group-box protein 1 and soluble hemoglobin scavenger receptor in brucellosis. Microbiol Immunol. 2013;57: $150-8$.

9. Sun $H$, Jiang $R$, Han $B, D u X, M a ~ C, X u Y$, et al. Serum levels of soluble CD163 and soluble CD14 following antibiotic therapy of patients with acute brucellosis. J Infect Dev Ctries. 2019;13:714-9.

10. Rubin LA, Galli F, Greene WC, Nelson DL, Jay G. The molecular basis for the generation of the human soluble interleukin 2 receptor. Cytokine. 1990;2: 330-6.

11. Yang ZZ, Grote DM, Ziesmer SC, Manske MK, Witzig TE, Novak AJ, et al. Soluble IL-2Ra facilitates IL-2-mediated immune responses and predicts reduced survival in follicular B-cell non-Hodgkin lymphoma. Blood. 2011; 118:2809-20.

12. Boyman $\mathrm{O}$, Sprent J. The role of interleukin-2 during homeostasis and activation of the immune system. Nat Rev Immunol. 2012;12:180-90.

13. Downes K, Marcovecchio ML, Clarke P, Cooper JD, Ferreira RC, Howson JM, et al. Plasma concentrations of soluble IL-2 receptor a (CD25) are increased in type 1 diabetes and associated with reduced C-peptide levels in young patients. Diabetologia. 2014;57:366-72.

14. Cabrera R, Ararat M, Cao M, Xu Y, Wasserfall C, Atkinson MA, et al. Hepatocellular carcinoma immunopathogenesis: clinical evidence for global T cell defects and an immunomodulatory role for soluble CD25 (sCD25). Dig Dis Sci. 2010;55:484-95.

15. Porcel JM, Gázquez I, Vives M, Pérez B, Rubio M, Rivas MC. Diagnosis of tuberculous pleuritis by the measurement of soluble interleukin 2 receptor in pleural fluid. Int J Tuberc Lung Dis. 2000;4:975-9.

16. Shitrit D, Izbicki G, Bar-Gil Shitrit A, Raz M, Sulkes J, Kramer MR. Role of soluble interleukin-2 receptor levels in patients with latent tuberculosis. Lung. 2006;184:21-4.

17. Novikov W, Egorova NI, Kurnikov GY, Evsegneeva IV, Baryshnikov AY, Karaulov AV. Serum levels of soluble HLA and IL-2R molecules in patients with urogenital chlamydia infection. Adv Exp Med Biol. 2007;601:285-9.

18. Makis AC, Galanakis E, Hatzimichael EC, Papadopoulou ZL, Siamopoulou A, Bourantas KL. Serum levels of soluble interleukin-2 receptor alpha (sIL-2Ralpha) as a predictor of outcome in brucellosis. J Inf Secur. 2005:51:206-10.

19. Tonna I, Tonna A. Brucellosis. N Engl J Med. 2005;353:1071-2 author reply 1071-2.

20. Goenka R, Parent MA, Elzer PH, Baldwin CL. B cell-deficient mice display markedly enhanced resistance to the intracellular bacterium Brucella abortus. J Infect Dis. 2011;203:1136-46.

21. Durward M, Radhakrishnan G, Harms J, Bareiss C, Magnani D, Splitter GA. Active evasion of CTL mediated killing and low quality responding CD8+ T cells contribute to persistence of brucellosis. PLoS One. 2012;7: e34925.

22. Durward-Diioia M, Harms J, Khan M, Hall C, Smith JA, Splitter GA. CD8+ T cell exhaustion, suppressed gamma interferon production, and delayed memory response induced by chronic Brucella melitensis infection. Infect Immun. 2015:83:4759-71.

23. Scharf O, Agranovich I, Lee K, Eller NL, Levy L, Inman J, et al. Ontogeny of Th1 memory responses against a Brucella abortus conjugate. Infect Immun. 2001;69:5417-22.

24. Galanakis E, Makis A, Bourantas KL, Papadopoulou ZL. Interleukin-3 and interleukin-4 in childhood brucellosis. Infection. 2002;30:33-4.

25. Annunziato F, Romagnani C, Romagnani S. The 3 major types of innate and adaptive cell-mediated effector immunity. J Allergy Clin Immunol. 2015;135: 626-35

26. Xavier MN, Winter MG, Spees AM, Nguyen $K$, Atluri $V L$, Silva TM, et al. CD4+ T cell-derived IL-10 promotes Brucella abortus persistence via modulation of macrophage function. PLoS Pathog. 2013;9:e1003454.

27. Pasquali $P$, Thornton AM, Vendetti S, Pistoia C, Petrucci $P$, Tarantino M, et al. CD4+CD25+ T regulatory cells limit effector $T$ cells and favor the progression of brucellosis in BALB/c mice. Microbes Infect. 2010;12:3-10.

28. von Bergwelt-Baildon MS, Popov A, Saric T, Chemnitz J, Classen S, Stoffel MS, et al. CD25 and indoleamine 2,3-dioxygenase are up-regulated by prostaglandin E2 and expressed by tumor-associated dendritic cells in vivo: additional mechanisms of T-cell inhibition. Blood. 2006;108:228-37.

29. Lindqvist CA, Christiansson LH, Simonsson B, Enblad G, Olsson-Strömberg U, Loskog AS. T regulatory cells control T-cell proliferation partly by the release of soluble CD25 in patients with B-cell malignancies. Immunology. 2010;131: $371-6$. 
30. Lienhardt C, Azzurri A, Amedei A, Fielding K, Sillah J, Sow OY, et al. Active tuberculosis in Africa is associated with reduced Th1 and increased Th2 activity in vivo. Eur J Immunol. 2002;32:1605-13.

31. Handzel ZT, Barak V, Altman Y, Bibi H, Lidgi M, lancovici-Kidon M, et al. Increased Th1 and Th2 type cytokine production in patients with active tuberculosis. Isr Med Assoc J. 2007:9:479-83.

32. Rodríguez-Zapata M, Matías MJ, Prieto A, Jonde MA, Monserrat J, Sánchez L, et al. Human brucellosis is characterized by an intense Th1 profile associated with a defective monocyte function. Infect Immun. 2010;78: 3272-9.

33. Rafiei A, Ardestani SK, Kariminia A, Keyhani A, Mohraz M, Amirkhani A. Dominant Th1 cytokine production in early onset of human brucellosis followed by switching towards Th2 along prolongation of disease. J Inf Secur. 2006;53:315-24.

34. Rittig MG, Kaufmann A, Robins A, Shaw B, Sprenger H, Gemsa D, et al. Smooth and rough lipopolysaccharide phenotypes of Brucella induce different intracellular trafficking and cytokine/chemokine release in human monocytes. J Leukoc Biol. 2003;74:1045-55.

35. Barquero-Calvo E, Chaves-Olarte E, Weiss DS, Guzmán-Verri C, Chacón-Díaz C, Rucavado A, et al. Brucella abortus uses a stealthy strategy to avoid activation of the innate immune system during the onset of infection. PLoS One. 2007:2:e631.

36. Barrionuevo P, Delpino MV, Pozner RG, Velásquez LN, Cassataro J, Giambartolomei GH. Brucella abortus induces intracellular retention of $\mathrm{MHC}-$ molecules in human macrophages down-modulating cytotoxic CD8(+) T cell responses. Cell Microbiol. 2013;15:487-502.

37. Hasanjani Roushan MR, Bayani M, Soleimani Amiri S, Mohammadnia-Afrouzi M, Nouri HR, Ebrahimpour S. Evaluation of CD4+ CD25+ FoxP3+ regulatory T cells during treatment of patients with brucellosis. J Biol Regul Homeost Agents. 2016;30:675-82.

Ready to submit your research? Choose BMC and benefit from:

- fast, convenient online submission

- thorough peer review by experienced researchers in your field

- rapid publication on acceptance

- support for research data, including large and complex data types

- gold Open Access which fosters wider collaboration and increased citations

- maximum visibility for your research: over $100 \mathrm{M}$ website views per year

At $\mathrm{BMC}$, research is always in progress.

Learn more biomedcentral.com/submissions 